\title{
Actividad antioxidante y antimicrobiana de extractos metanólicos de hojas de plantas del género Solanum
}

\author{
José A. Ramón-Valderrama ${ }^{1,2}$ y Paula L. Galeano-García ${ }^{1,2 *}$ \\ (1) Facultad de Ciencias Básicas, Programa de Química, Universidad de la Amazonia Calle 17 Dg 17 Cra $3 F$, Barrio \\ Porvenir, Florencia, Colombia (correo-e: ramonaries04@gmail.com, \\ (2) Grupo de Investigación en Productos Naturales Amazónicos, Universidad de la Amazonia, Florencia, Colombia \\ (correo-e: p.galeano@udla.edu.co)
}

${ }^{*}$ Autor a quien debe ser dirigida la correspondencia.

Recibido Abr. 18, 2019; Aceptado Jun. 9, 2020; Versión final Ago. 5, 2020, Publicado Oct. 2020

\begin{abstract}
Resumen
En esta investigación se evaluó la actividad antioxidante y antimicrobiana de los extractos metanólicos de diez especies del género Solanum. A través de modelos in vitro se determinó la actividad antioxidante, el contenido de fenoles y flavonoides totales. La presencia de fenoles totales se evaluó por cromatografía líquida de alta resolución y la actividad antimicrobiana se evaluó usando los microorganismos Klebsiella pneumoniae, Staphylococcus aureus y Escherichia coli. Los resultados mostraron variaciones en el contenido de fenoles totales (54 a $286 \mathrm{mg}$ eq. Ac gálico/g) y flavonoides totales (18 a $73 \mathrm{mg}$ eq. quercetina/g). Se evidenció variaciones en actividad antiradical DPPH $(0,01$ a $5,2 \mu \mathrm{mol}$ eq. Trolox $/ 100 \mathrm{~g})$, ABTS ${ }^{++}(0,03 \mathrm{a} 2,01 \mu \mathrm{mol}$ eq. Trolox/100 g) y reductora FRAP (202 a $887 \mu \mathrm{mol}$ eq. Ac. ascórbico/g). Los extractos S. mammosum y $S$. barbeyanum presentaron actividad antimicrobiana frente a Escherichia coli. Se concluye que el extracto $S$. mammosum es una fuente principal de compuestos bioactivos.
\end{abstract}

Palabras clave: antioxidantes; antimicrobiana; compuestos fenólicos; Solanaceae; Solanum

\section{Antioxidant and antimicrobial activities in leaf methanolic extracts from the plant genus Solanum}

\begin{abstract}
The present research study assessed antioxidant and antimicrobial activities in leaf methanolic extracts from ten plant species of the genus Solanum. Antioxidant activity (phenol content and total flavonoids) was determined by using in vitro models. The presence of total phenols was evaluated by high-performance liquid chromatography. Antimicrobial activity was examined by using the microorganisms Klebsiella pneumoniae, Staphylococcus aureus, and Escherichia coli. The results showed variations in total phenol content (54 to 286 $\mathrm{mg}$ eq. gallic $\mathrm{Ac} / \mathrm{g}$ ) and total flavonoids (18 to $73 \mathrm{mg}$ eq. quercetin/g). There were variations in anti-radical DPPH activity (0.01 to $5.2 \mu \mathrm{mol}$ eq. Trolox/100 g), ABTS ${ }^{++}(0.03$ to $2.01-\mu \mathrm{mol}$ eq. Trolox/100 g), and FRAP reducer (202 to $887 \mu \mathrm{mol}$ eq. Ac. ascorbic/g). Both S. mammosum and S. barbeyanum extracts showed antimicrobial activity against Escherichia coli. It is concluded that the $S$. mammosum extract is a major source of bioactive compounds.
\end{abstract}




\section{INTRODUCCIÓN}

La familia Solanaceae, una de las más grandes y complejas de las angiospermas, incluye 2500 especies en 100 géneros (Almoulah et al; 2017). Estas plantas exhiben un amplio espectro de entidades químicas con diferentes actividades biológicas, importantes desde el punto de vista económico, agrícola y farmacéutico (Zadra et al., 2012). El género Solanum es uno de los más grandes de la familia de las Solanaceae, posee alrededor de 1700 especies distribuidas en todo el mundo (Siddiqui et al; 2017). Este género presenta gran riqueza y diversidad de propiedades farmacológicas; entre ellas, actividad citotóxica, anticancerígena, antiinflamatoria, antiulcerogénica, antimicrobiana y antioxidante (Costa et al; 2018). Algunos reportes indican que S. mammosum es una fuente principal de Solasodina, un alcaloide conocido por sus efectos diuréticos, anticancerígenos, antifúngicos, cardiotónicos, antiespermatogenéticos, antiandrogénicos, inmunomoduladores y antipiréticos en el sistema nervioso central (Patel et al., 2013). De otro lado se reporta la actividad antioxidante frente a los radicales libres $\mathrm{ROO},{ }^{\circ} \mathrm{OH}$ y ONOO de extractos acuosos de frutos de $S$. sessiliflorum Dunal atribuido a la presencia de compuestos carotenoides como el $\beta$-caroteno y luteína $(\beta, \varepsilon$-caroteno-3,3'diol), y compuestos fenólicos como ácido clorogénico (ácido 5-O-cafeoil-ácido quínico) (Rodríguez et al; 2013). Esta propiedad antioxidante puede relacionarse a otros componentes como, alcaloides, glicósidos, flavonoides, cumarinas, antocianinas y taninos (Mascato et al. 2015). Así mismo, tiene efecto inhibitorio in vitro frente a Helicobacter pylori (Pardo, 2010).

De igual forma, los frutos de $S$. quitoense Lam. contienen carotenoides (trans-beta-caroteno, 13-cis-betacaroteno, 9-cis-beta-caroteno y luteína), ácido ascórbico y compuestos fenólicos con propiedades antioxidante y antimicrobiana (Gancel et al; 2008). En cuanto a frutos de S. grandiflorum se indicó la presencia de flavonoides y compuestos fenólicos con actividad antioxidante y actividad fungicida frente a Rhizoctonia solani (Ghassam et al; 2012). Adicionalmente, se reportó que los extractos alcohólicos de hojas de S. hartwegii Benth contienen compuestos como alcaloides, flavonoides y antocianinas. Del mismo modo, estos metabolitos secundarios fueron encontrados en extractos etanólicos de hojas de S. jamaicense Mill, adicionalmente, triterpenos, saponinas, taninos y cumarinas que representan actividad fungicida frente a Candida albicans (Anselmo y Lima, 2014). Por la importancia que tiene la familia Solanaceae y particularmente el género Solanum, y la poca información relacionada a sus componentes fenólicos, actividad antioxidante y antimicrobiana, el presente estudio tiene como propósito, evaluar dichas propiedades en 10 extractos metanólicos de hojas de especies del género Solanum.

\section{MATERIALES Y MÉTODOS}

Para las mediciones colorimétricas y cuantitativas se usaron como instrumentos; espectrofotómetro Genesys 5.0 y cromatógrafo líquido Shimadzu LC 2010HT. Por cada experimento se realizaron 4 réplicas.

\section{Material vegetal}

Las hojas de 10 especies de Solanum (Solanaceae) fueron recolectadas en el municipio de Florencia (Caquetá), durante los meses marzo y abril. Lo puntos de colecta fueron: 1) centro de investigaciones Macagual ( $\left.1^{\circ} 37^{\prime} \mathrm{N}-75^{\circ} 36^{\prime} \mathrm{W}\right)$; 2) granja Balcanes ( $01^{\circ} 25^{\prime} 5,75^{\prime \prime} \mathrm{N}-075^{\circ} 30^{\prime} 9,72^{\prime \prime} \mathrm{W}$ ) y 3) km 47 vía Suaza (Huila) $\left(1^{\circ} 45^{\prime} 15^{\prime \prime} \mathrm{N}-75^{\circ} 41^{\prime} 36^{\prime \prime} \mathrm{W}\right)$. Los ejemplares de cada especie se llevaron al herbario de la Universidad de la Amazonia, Uniamazonia-Huaz, registradas en diferentes números de váucher; $S$. mammosum: 014286; $S$. senssiliflorum: 014277; S. grandiflorum: 014279; S. quitoense: 014285; S. candidum: 014281; S. rugosum: 014278; S. jamaicense: 014282; S. barbeyanum: 014280; S. schlechtendalianum: 014284 y S. hartwegli: 014283. Las hojas se colectaron sanas, se deshidrataron en un horno a $45^{\circ} \mathrm{C}$ por 72 horas y luego se maceraron en un molino.

\section{Obtención de los extractos metanólicos de Solanum (EMS)}

Se tomaron aproximadamente $35 \mathrm{~g}$ de hojas maceradas de cada especie vegetal, suspendidas en $120 \mathrm{~mL}$ de $\mathrm{MeOH}(99 \mathrm{v} / \mathrm{v})$ durante 3 días. Después la biomasa fue filtrada al vacío, y el residuo insoluble se re-extrajo 2 veces consecutivas, con igual proporción de solvente. Los sobrenadantes de cada extracción fueron concentrados a presión reducida a $45^{\circ} \mathrm{C}$ en un rota-evaporador (Marca Heidolph) hasta sequedad (extracto crudo) para determinar su rendimiento. Los 10 extractos secos de Solanum (EMS) fueron re-suspendidos en metanol a una concentración de $10 \mathrm{mg} / \mathrm{mL}$ para los análisis posteriores.

\section{Ensayo de decoloración del radical (DPPH')}

La actividad antioxidante se evaluó por el método de DPPH', 2,2-difenil-1-picrilhidracilo (25,4 mM, ajustada con metanol a 0,3 unidades de absorbancia), según la metodología descrita por Williams et al; 1995. Para el ensayo se mezcló $10 \mu \mathrm{L}$ de cada extracto a concentraciones diferenciadas entre 50 a $12500 \mathrm{mg} / \mathrm{L}$ y $990 \mu \mathrm{L}$ de DPPH. Luego de $30 \mathrm{~min}$ en la oscuridad se midió la absorbancia a una longitud de onda de $517 \mathrm{~nm}$. Los resultados se expresaron como valores TEAC, considerando una curva patrón de TROLOX® $(0,1-1,0 \mathrm{mM}$, 
$\left.\mathrm{R}^{2}=0,998\right)$. El parámetro que se midió es el porcentaje de reducción del DPPH (\%lnh) frente a la muestra, ecuación (1), donde $A$ muestra es absorbancia de la muestra y $A_{\text {control }}$ es absorbancia del reactivo DPPH.

$$
\% \operatorname{Inh}=\left(1-\frac{A_{\text {muestra }}}{A_{\text {control }}}\right) * 100
$$

Por regresión lineal, se construyó curvas del porcentaje de inhibición vs concentración de las soluciones de Trolox y se calculó el IC50 en $\mu \mathrm{mol}$ eq. Trolox/L. Del mismo modo, se determinó el IC50 de los extractos vegetales expresados en $\mathrm{mg}$ de EMS/L, se calculó y expresó la capacidad antioxidante en $\mu \mathrm{mol}$ eq. Trolox por $100 \mathrm{~g}$ de extracto seco (TEAC), mediante la ecuación (2).

$$
\text { TEAC }=\frac{\text { IC50 Trolox }}{\text { IC50 EMS }}
$$

Donde IC50 Trolox, cantidad a la cual el antioxidante Trolox inhibe el $50 \%$ de los radicales libres presentes e IC50 EMS, cantidad a la cual los antioxidantes de los EMS inhiben el $50 \%$ de los radicales libres presentes

\section{Actividad inhibidora del radical (ABTS)}

El método ABTS', 2,2'-azino-bis (3-etilbenzotiazolin)-6-sulfonato de amonio (3,5 mM, ajustada con metanol a 0,7 unidades de absorbancia), se desarrolló según la metodología descrita por Fellegrini et al; 1999. Para el ensayo se combinó $10 \mu \mathrm{L}$ de EMS a concentraciones variantes entre 405 a $5300 \mathrm{mg} / \mathrm{L}$ y $990 \mu \mathrm{L}$ de ABTS: Después de 30 min se leyó el cambio en la absorbancia a $730 \mathrm{~nm}$ (longitud de onda de máxima absorción). Los datos se expresaron como valores TEAC ( $\mu \mathrm{mol}$ eq. Trolox por $100 \mathrm{~g}$ de extracto seco), considerando una curva patrón de TROLOX® $\left(0,1-1,0 \mathrm{mM}, \mathrm{R}^{2}=0,998\right)$.

\section{FRAP}

El ensayo se realizó según la metodología propuesta por Benzie y Strain (1996). Se mezcló $900 \mu \mathrm{L}$ de FRAP (buffer ácido acético-acetato de sodio pH 3.4, TPTZ (2, 4,6-tripiridil-s-triazina, 1,9 mM) y FeCl 3 ); $50 \mu \mathrm{L}$ de cada extracto a concentraciones diferencias entre 1000 a $6000 \mathrm{mg} / \mathrm{L}$ y $50 \mu \mathrm{L}$ de buffer acetato. Después de $30 \mathrm{~min}$ se midió la máxima absorbancia del $\mathrm{Fe}^{2+}$ a $590 \mathrm{~nm}$. Los valores se expresaron como FRAP ( $\mu \mathrm{mol}$ eq. Ac. ascórbico/g de EMS) mediante una curva patrón de ácido ascórbico (100-800 mg eq. Ac. ascórbico/L, $\left.\mathrm{R}^{2}=0,999\right)$.

\section{CFT (Contenido de Fenoles Totales)}

El CFT se determinó mediante el método de Folin-Ciocalteu propuesto por Singleton y Rossi (1965) con modificaciones. El ensayo consistió en hacer reaccionar $60 \mu \mathrm{L}$ de EMS a concentraciones variantes entre 1200 a $5500 \mathrm{mg} / \mathrm{L} ; 415 \mu \mathrm{L}$ de agua y $125 \mu \mathrm{L}$ de reactivo Folin-Ciocalteau. Luego se adicionó $400 \mu \mathrm{L}$ de $\mathrm{Na}_{2} \mathrm{CO}_{3}(7,1 \%)$. La reacción se dejó en reposo por $30 \mathrm{~min}$ y se midió la absorbancia a $760 \mathrm{~nm}$. Los valores se expresaron en mg eq. Ac. gálico/g de EMS mediante una curva patrón de ácido gálico (100-600 mg eq. Ac. gálico/L, $R^{2}=0,998$ ).

\section{FVT (Flavonoides Totales)}

Los FVT se determinaron por el método de formación del complejo $\mathrm{AICl}_{3}$ descrito por Zhishen et al; 1999 con modificación en la metodología. Se mezcló $400 \mu \mathrm{L}$ de agua destilada, $100 \mu \mathrm{L}$ de EMS con concentraciones entre 1500 a $4500 \mathrm{mg} / \mathrm{L}, 30 \mu \mathrm{L}$ de una solución de $\mathrm{NaNO}_{2}(5 \%)$ y se homogeneizó. Después de 5 min se adicionó $30 \mu \mathrm{L}$ de una solución de $\mathrm{AlCl}_{3}(10 \%)$. Luego se agregó $200 \mu \mathrm{L}$ de una solución de $\mathrm{NaOH}(1 \%)$ y se dejó reposar por 15 min y por último se adicionó $240 \mu \mathrm{L}$ de agua destilada. Después se midió la absorbancia a $510 \mathrm{~nm}$. Los resultados se expresaron como (mg eq. quercetina/g de EMS) mediante una curva patrón de quercetina $\left(5-150 \mathrm{mg} / \mathrm{L}, \mathrm{R}^{2}=0,997\right)$.

\section{Análisis de FVT por Cromatografía Líquida de Alta Resolución (HPLC)}

La separación cromatográfica de los FVT presentes en los EMS se realizó en un equipo HPLC con capacidad de hacer gradiente de eluyentes, auto muestreador, bomba cuaternaria, desgasificador y detector UV. Para la separación se usó una columna C18 (180 mm x 4,6 mm i.d x $5 \mu \mathrm{m})$, sistema disolvente de fase móvil; agua: $\mathrm{MeOH} 55: 45(\mathrm{v} / \mathrm{v})$, volumen de inyección $5 \mu \mathrm{L}$, velocidad de flujo $0,5 \mathrm{~mL}^{\mathrm{min}}{ }^{-1}$, temperatura $40^{\circ} \mathrm{C}$ y longitud de onda de $280 \mathrm{~nm}$. 


\section{Actividad antimicrobiana}

Para los ensayos se usaron las cepas bacterianas Klebsiella pneumoniae (ATCC 70603), Staphylococcus aureus (ATCC 25923) y Escherichia coli (ATCC 25922) adquiridas en el Instituto Tecnológico de Biotecnología de la Universidad Nacional de Colombia. Este ensayo rápido tipo Screaning, consideró el método de difusión en agar con sensidisco propuesto por Mora et al; 2007. Se usó agar Müller-Hinton como cultivo microbiológico $\left(\mathrm{pH} 7.2-7.6 ; 20 \mathrm{~g} / \mathrm{L}, 37^{\circ} \mathrm{C}\right.$ ) con previa esterilización. A partir de cultivos de las cepas bacterianas en caldo (24 horas, $24 \pm 2^{\circ} \mathrm{C}$ ), se ajustó la concentración de inóculo de acuerdo al patrón de turbidez № 0,5 de Mc Farland. Los sensidiscos (5,2 mm de diámetro) fueron impregnados con $20 \mathrm{mg} / \mathrm{mL}$ de los EMS (100 $\mu \mathrm{l})$, colocados sobre cajas de Petri expuestos a los microorganismos mencionados, como control positivo se usó tetraciclina y control negativo Dimetilsulfóxido. Las cajas fueron incubadas a $24 \pm 1 \stackrel{\circ}{ } \mathrm{C}$ por 24 horas, después se midieron los diámetros de los halos formados usando una tabla de medición específica, corroborando estas lecturas a las 48 horas de incubación.

\section{Análisis estadístico}

Para el análisis de componentes principales se usó el software Excel, XLSTAT, la comparación de medias (prueba de rango múltiples de Duncan con un nivel de significancia del $95 \%, p<0,05$ ) y correlación de Pearson se usó el programa InfoStat Versión Estudiantil, 2016.

\section{RESULTADOS Y DISCUSIÓN}

Se evalúo el potencial antioxidante y antibacteriano de los extractos metanólicos de hojas de 10 especies de plantas del género Solanum. La figura 1, muestra las 10 especies estudiadas; entre las cuales, los frutos de S. senssiliflorum, S. quitoense y S. candidum son comestibles.

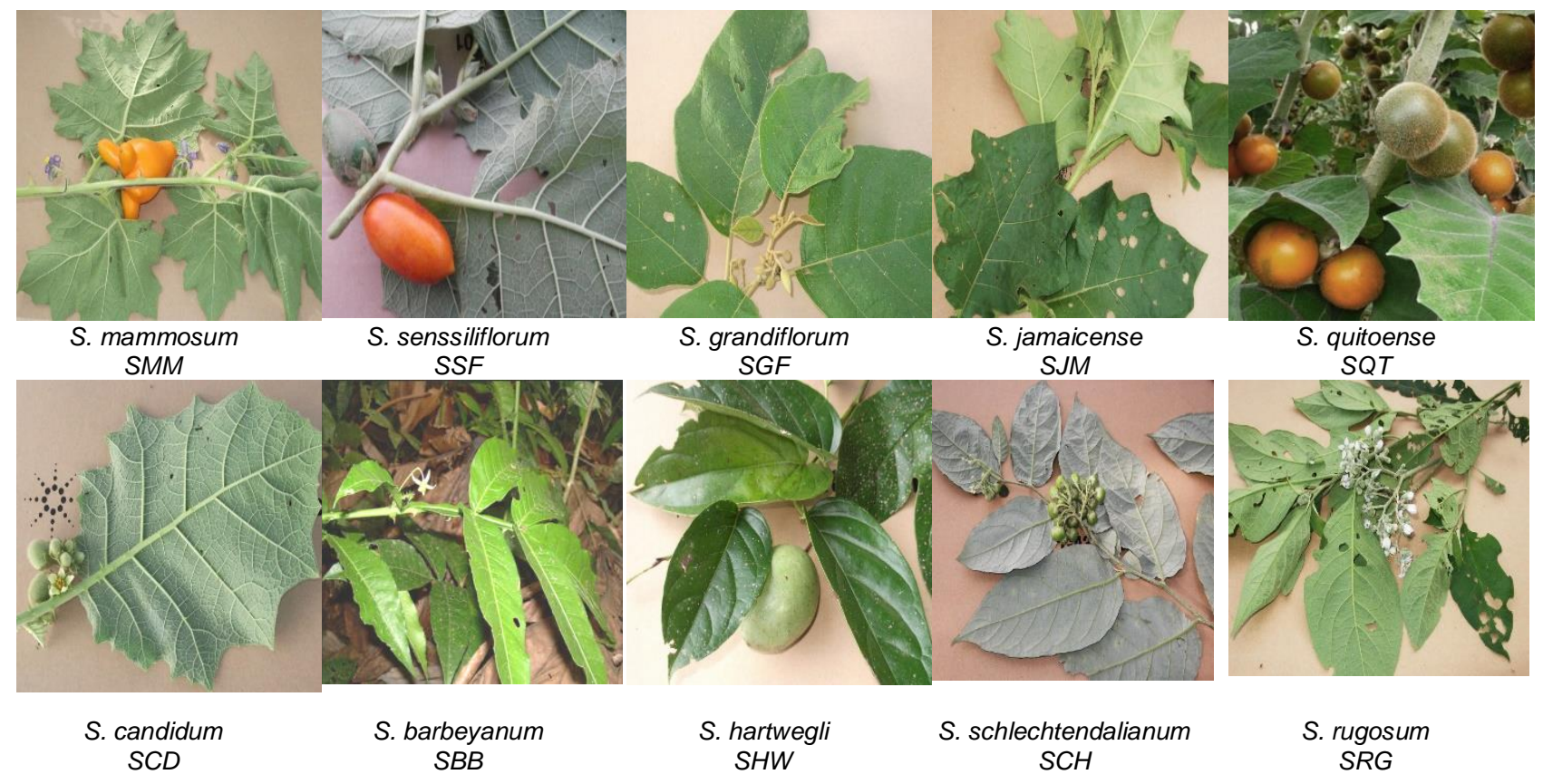

Fig. 1: Imágenes fotográficas de las especies de Solanum estudiadas

Los EMS presentaron valores de CFT (54 \pm 1 a $286 \pm 8 \mathrm{mg}$ eq. Ac. gálico/g de muestra seca) y FVT (18 \pm 1 a $73 \pm 2 \mathrm{mg}$ eq. Quercetina/g de muestra seca); según la comparación de medias (prueba de rango múltiples de Duncan con un nivel de significancia del $95 \%$, los extractos SMM y SCH difieren estadísticamente de los demás en cuando al contenido de fenoles y flavonoides totales, siendo el extracto S. mammosum (SMM) con mayor contenido de FVT y CFT. Así mismo, una capacidad reductora del ion $\mathrm{Fe}^{3+}$ con valores que oscilaron entre 202 a $887 \mu \mathrm{mol}$ eq. Ac. ascórbico/g (Fig.2). Del mismo modo, el potencial antioxidante de los EMS evaluado a través de modelos in vitro mostró una actividad antiradical DPPH con valores TEAC entre 0,01 \pm $0,1 \mathrm{a} 5,2 \pm 0,4 \mu \mathrm{mol}$ eq. Trolox/ $100 \mathrm{~g}$ de EMS seco y ABTS ${ }^{*+}$ entre $0,03 \pm 1$ a $2,01 \pm 1 \mu \mathrm{mol}$ eq. Trolox/100g de EMS seco (Fig. 3). Los extractos vegetales SQT, SCD, SBB y SRG no son significativamente diferentes ( $p$ $>0,05$ ), en cuanto a su capacidad DPPH. Sin embargo, son estadísticamente diferentes en cuanto la actividad FRAP. De igual forma, el extracto $S$. mammosum fue el que presentó los máximos valores de actividad antioxidante. 
En este estudio los 10 extractos metanólicos del género Solanum mostraron alto contenido fenólico evaluado por las metodologías de CFT y FVT, lo cual indicaría que las especies de Solanum presentan compuestos activos que pueden detener la oxidación de lípidos (Zamora y Hidalgo, 2016), inhibir reacciones de quimioluminiscencia (Haddabi et al; 2017), capturar especies reactivas de oxígeno (Saw et al; 2014) y actuar como donantes de hidrógeno o electrones (Kaewnarin et al; 2016). En cuanto a la actividad antiradical DPPH• y $\mathrm{ABTS}^{++}$de los EMS puede deberse a la presencia de compuestos activos que poseen la capacidad de donar hidrógenos o electrones; esenciales para la captura de especies reactivas de oxígeno. Del mismo modo, los valores FRAP se constituyen en extractos capaces de reducir $\mathrm{Fe}^{3+}$ mediante transferencia de electrones. Los compuestos fenólicos presentan actividad antioxidante debido a su estructura y peso molecular y número de oxidrilos sustituyentes (Xie et al; 2015).

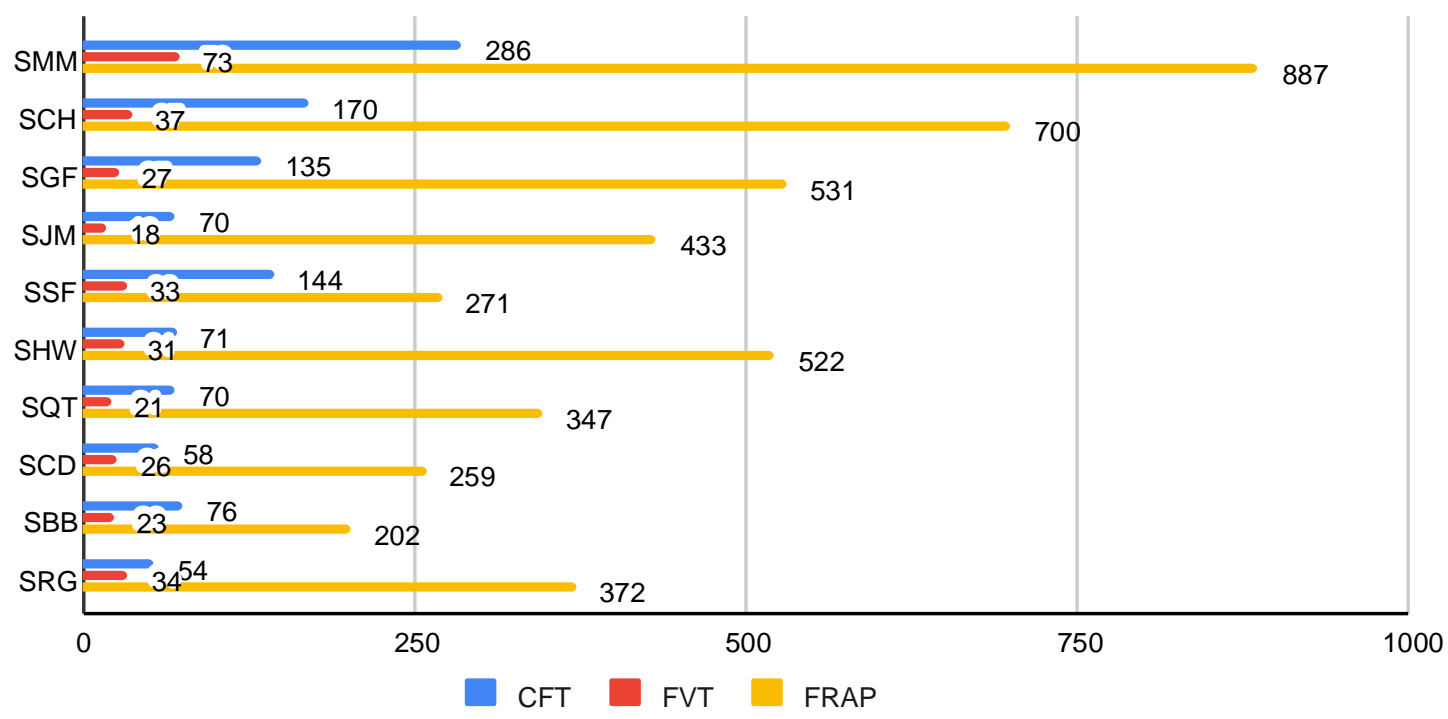

Fig. 2: Contenido de fenoles totales, flavonoides totales y actividad FRAP de los EMS estudiados

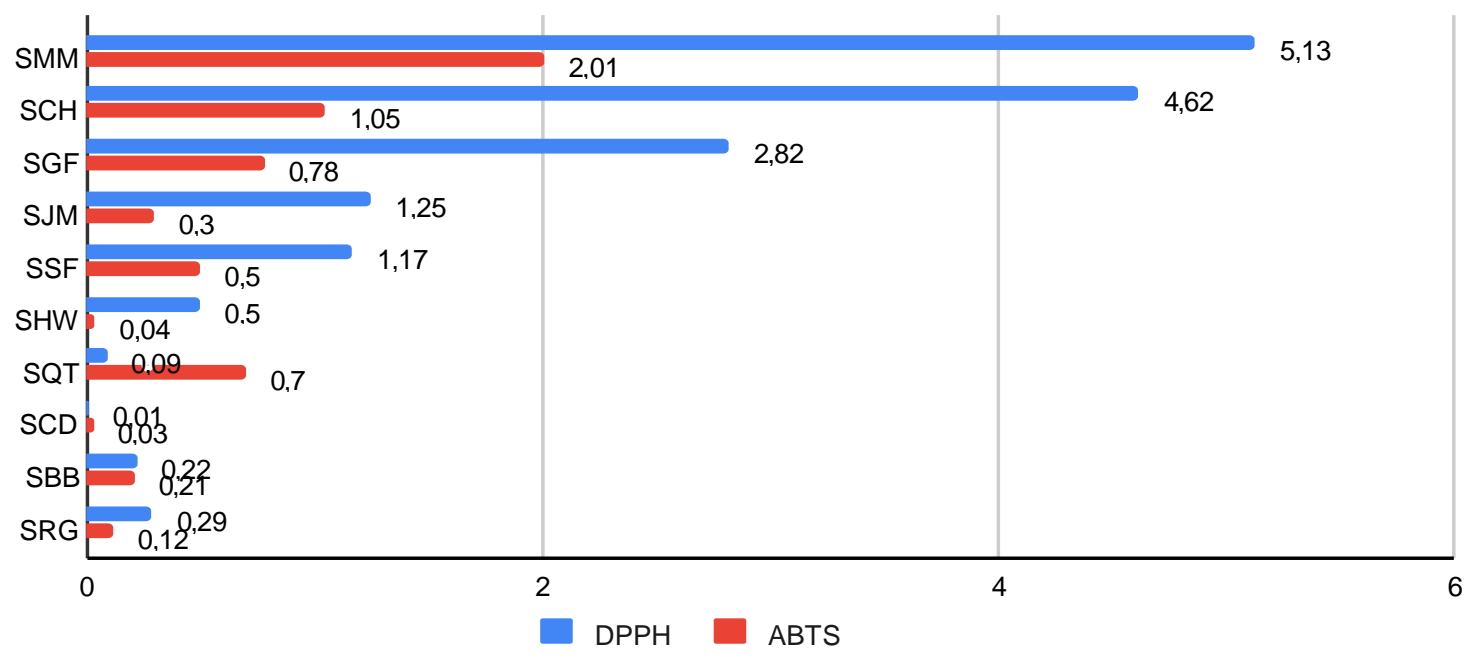

Fig.3: Capacidad antiradical DPPH y ABTS de los EMS estudiados, expresada en valores TEAC

En la actualidad no hay reportes sobre composición fenólica y actividad antioxidante en hojas de las especies de Solanum evaluadas. Sin embargo, se ha reportado actividad antioxidante en frutos de $S$. senssiliflorum Dunal (Rodríguez et al; 2013), S. quitoense Lam (Gancel et al; 2008) y S. grandiflorum (Ghassam et al; 2012), la cual es asociada a la presencia de compuestos fenólicos como flavonoides, principalmente antocianinas; ácidos fenólicos y taninos. Del mismo modo, en otras especies como S. melongena (Nisha et al; 2009) y $S$. lycopersicum ( $\mathrm{Li}$ et al; 2014) se reportaron contenidos fenólicos de 106,98 y 659,25 mg eq. Ac. gálico/g, respectivamente; actividad ABTS y FRAP de 166,99 $\mu \mathrm{mol}$ eq. Trolox/g y $68,9 \mu \mathrm{mol}$ eq. Ac. ascórbico/g, respectivamente. Por otro lado, en extractos de cáscara de $S$. melongena $L$ se reportó una capacidad antioxidante en un rango de IC50 de 8.42 a $37.28 \mathrm{mg} / \mathrm{L}$ (Heras y Alvis, 2013).

En hojas de $S$. surttense se reportó un contenido fenólico de $46,7 \mathrm{mg}$ eq. Ac. gálico/g y actividad antiradical ABTS de 89,28 $\mu \mathrm{mol}$ eq. Trolox/g (Murahan et al; 2013). Igualmente, en hojas de S. nigrum L, Cai et al; 2004 reportaron un contenido de CFT de $220 \mathrm{mg}$ eq. Ac. gálico/g y actividad ABTS de 46,7 $\mu \mathrm{mol}$ eq. Trolox/g. En extractos secos de hojas de S. macrocarpon se determinó CFT de 421,09 mg eq. Ac. gálico/ g, DPPH 23,81 
mg eq. Ac. ascórbico / g y FRAP 56.46 mg eq. Ac. ascórbico/ g (Famuwagun et al; 2017). Finalmente, en extractos de hojas de la especie $S$. schimperianum se informó una actividad antioxidante con valores de DPPH IC 50 de 3,5 $\pm 0,2$ y ABTS de 3,5 $\pm 0,3 \mu \mathrm{g} / \mathrm{mL}$ (Fadl et al; 2017). Estos valores de actividad y composición fenólica en frutos y hojas de otras especies son inferiores a los encontrados en $S$. mammosum y $S$. schlechtendalianum.

Según el análisis de componentes principales (ACP) sobre la matriz de correlación de las variables cuantitativas; actividad antioxidante (DPPH, FRAP, ABTS), contenido fenólico (CFT, FVT), tiempo de retención (TR) y altura de pico (flavonoides detectados a $280 \mathrm{~nm}$ ), y las dos primeras coordenadas del análisis de coordenadas principales (ACoorP) de los extractos vegetales (PCO1 y PCO2), explicó para los dos primeros ejes el $76,7 \%$ de la variabilidad acumulada total (Fig. 4). Como puede observarse el primer componente principal (F1) explicó el $62,2 \%$ de la variabilidad, separando SMM, SCH y SGF de los demás extractos vegetales, por lo tanto, la mayor variabilidad en cuanto al contenido fenólico, actividad antioxidante y abundancia de compuestos fenólicos es explicada en estos tres extractos; esto es debido a sus características farmacológicas de sus compuestos.

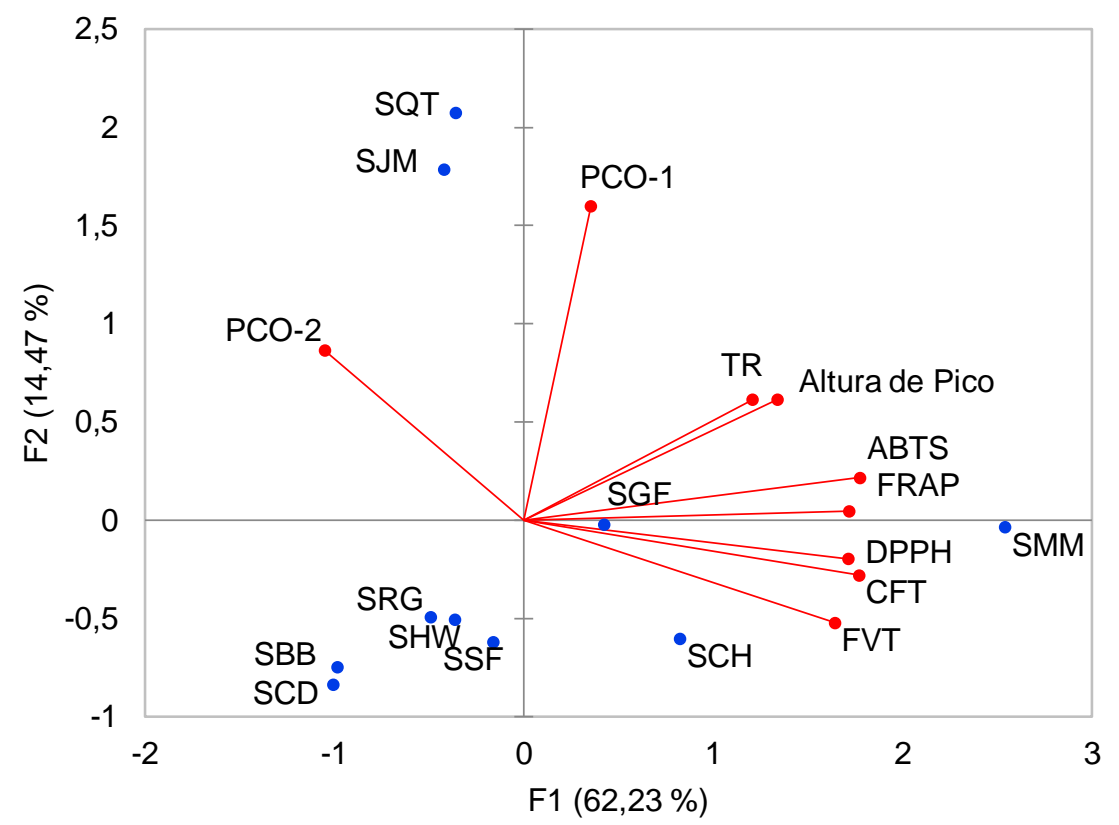

- Variables activas - Extractos Vegetales

Fig. 4: Biplot, análisis de componentes principales y el ACoorP (PCO1 y PCO2) sobre la matriz actividad antioxidante, contenido de fenoles y flavonoides totales; y datos HPLC (altura de pico y tiempo de retención)

Los compuestos fenólicos exhiben máximos de absorbancia en la región de 275-285 nm debido al anillo aromático en su estructura molecular. Los ácidos hidroxibenzoicos se detectan a $280 \mathrm{~nm}$ y las flavanonas en el rango de 277-295 nm con un hombro a 300-330 nm (Mascherpa et al; 2012). En este estudio se realizó un análisis por HPLC-UV para establecer la presencia de estos compuestos fenólicos en los extractos metanólicos a una longitud de onda de $280 \mathrm{~nm}$. En la Fig.5 presenta los perfiles cromatográficos de algunos extractos vegetales de Solanum estudiados, aunque solo se detectó un compuesto con mayor intensidad en $\mathrm{SMM}$ a un $\mathrm{T}_{\mathrm{R}}=1,41 \mathrm{~min}$ (Fig. $5 \mathrm{C}$ ), este le puede conferir propiedades farmacológicas a la especie. Del mismo modo, se destaca el extracto vegetal SCH con nueve compuestos fenólicos detectados en un rango de tiempo de retención entre 0,78 a 3,27 min (Fig. 5E), estos compuestos pueden aportar los efectos antioxidantes a la planta.

Para entender el mecanismo oxidativo de los EMS se realizaron correlaciones de Pearson entre las técnicas de actividad antioxidante y el contenido de compuestos fenólicos totales. Se presentaron valores de correlación positiva mayores a 0,80 . Se destacan los valores de correlaciones entre la actividad DPPH vs. CFT con $R^{2}=0,90$ y $A B T S$ vs. CFT con $R^{2}=0,94$. Así mismo, la correlación entre $D P P H$ vs $A B T S$ con $R^{2}=0,88$ y ABTS vs. FRAP con un $R^{2}=0,82$. La relación entre el contenido de fenoles y la actividad antioxidante establece que entre 0,80 y 0,94 de la actividad evaluada para los EMS de la región Andino-amazónica colombiana resulta de la contribución del contenido fenólico, son buenos antioxidantes (donan átomos de hidrógeno o electrones), y se expresan muy bien como agentes reductores. 

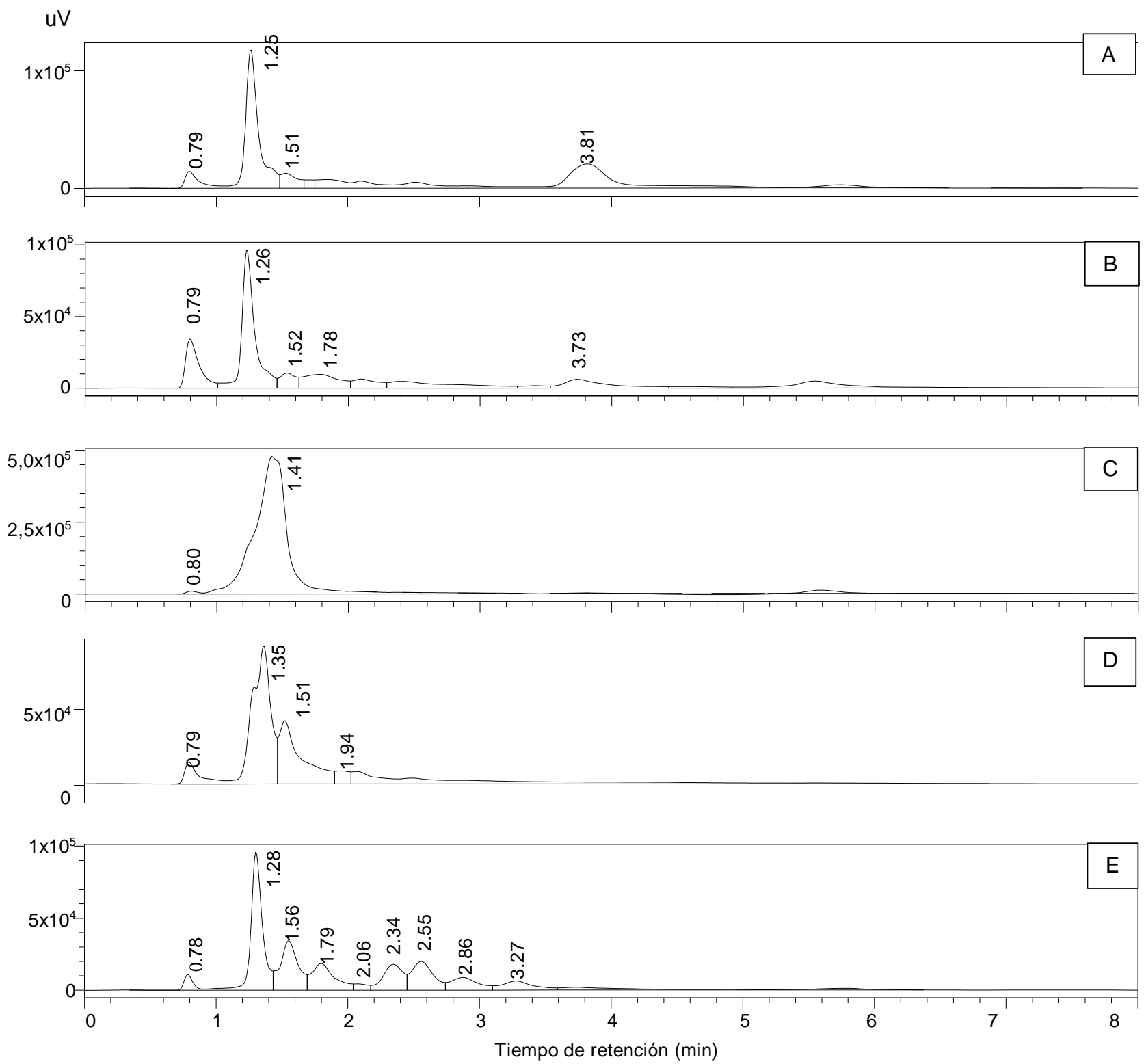

Fig. 5: Perfiles cromatográficos de los extractos vegetales de (A) SJM, (B) SSF, (C) SMM, (D) SGF y (E) SCH (TR 0-8 min), longitud de onda de detección $280 \mathrm{~nm}$

De otro lado, los ensayos de difusión en agar usados para evaluar la actividad antimicrobiana demostraron que el extracto $S$. mammosum (SMM) y $S$. barbeyanum (SBB) presentaron actividad antimicrobiana frente al microorganismo Escherichia coli a una concentración de $20 \mathrm{mg} / \mathrm{mL}$ (Tabla 1), lo cual predice la presencia de compuestos bioactivos con interés farmacológico (Cetin y Newman, 2015). Sin embargo, los estudios realizados en los 10 extractos de Solanum no inhiben el crecimiento de los microorganismos Klebsiella pneumoniae y Staphylococcus aureus.

Tabla 1. Actividad antimicrobiana de los EMS frente a K. pneumoniae, S. aureus y E. coli, los signos (+) Activo (1-2mm), $(+++)$ mayor actividad (3-6mm), (-) ausencia de actividad a una concentración $20 \mathrm{mg} / \mathrm{mL}$, TCN: tetraciclina, BC: blanco.

\begin{tabular}{|l|l|l|l|l|l|l|l|l|l|l|l|l|}
\hline Actividad & SMM & SCH & SGF & SJM & SSF & SHW & SQT & SCD & SBB & SRG & TCN & $B C$ \\
\hline S. aureus & - & - & - & - & - & - & - & - & - & - & +++ & - \\
\hline E. coli & + & - & - & - & - & - & - & - & + & - & +++ & - \\
\hline K. pneumoniae & - & - & - & - & - & - & - & - & - & - & +++ & - \\
\hline
\end{tabular}

En las especies estudiadas, el único reporte sobre análisis antimicrobiano lo presenta la especie $S$. hartwegli, según Morales, 2011 en el cual extractos de hojas de esta especie no inhiben el crecimiento microbiano de $S$. aureus y E. coli a concentraciones de $1 \mathrm{mg} / \mathrm{mL}$, lo cual es acorde a lo reportado en este trabajo. En otras especies del mismo género como $S$. schimperianum no inhibe el crecimiento de Staphylococcus aureus $y$ Escherichia coli a concentraciones de $10 \mu \mathrm{g} / \mathrm{mL}$ (Al-Rehaily et al; 2013). Sin embargo, Rawani et al; 2013, 
reportan que extractos de hojas de $S$. nigrum $L$ inhibe el crecimiento microbiano de Staphylococcus aureus a concentraciones de $10 \mathrm{mg} / \mathrm{mL}$.

\section{CONCLUSIONES}

De acuerdo a los resultados de este estudio los extractos vegetales de Solanum presentaron una correlación positiva entre la actividad antioxidante y el contenido de fenoles y flavonoides totales; por lo tanto, se concluye que los compuestos fenólicos presentes en estos extractos son capaces de capturar radicales libres y se pueden expresar como buenos agentes reductores. Según los resultados de actividad antioxidante, contenido fenólico y actividad antimicrobiana se propone la especie Solanum mammosum como fuente principal de compuestos bioactivos

\section{AGRADECIMIENTOS}

Los autores agradecen a la Universidad de la Amazonia y al programa de Jóvenes Investigadores, convocatoria No. 617-2013 "Semilleros-Jóvenes Investigadores, Doctorados Nacionales, en el Exterior e Inserción Laboral-Año 2013" de Colciencias.

\section{REFERENCIAS}

Al-Rehaily, A.J., Ahmad, M.S., y otros cinco autores, Solanopubamine a rare steroidal alkaloid from Solanum schimperianum: Synthesis of some new alkyl and acyl derivatives their anticancer and antimicrobial evaluation. doi: 10.1016/j.jscs.2011.10.003, Journal of Saudi Chemical Society, 17, 67-6 (2013).

Almoulah, N.F., Voynikoy, Y., y otros diez autores, Antibacterial, antiproliferative and antioxidant activity of leaf extracts of selected Solanaceae species. doi: 10.1016/j.sajb.2017.06.016, South African Journal of Botany 112(3), 368-374 (2017).

Anselmo, J y Lima, R., Identification of class the secondary metabolites in leaves extracte thanolic Solanum jamaicense (solanaceae) and its potential fungicide about candida albicans in vitro. doi: 10.5216/ref.v11i1.27632, Revista Electrónica de Farmacia, 11(1), 11-20 (2014).

Benzie, I., y Strain, J., The ferric reducing ability of plasma (FRAP) as ameasure of "Antioxidant Power": The FRAP assay. doi: 10.1006 / abio.1996.0292, Analytical Biochemistry, 239(1), 70-76 (1996).

Cai, Y., Luo, Q., Sun, M; y Corke, H., Antioxidant activity and phenolic compounds of 112 traditional Chinese medicinal plants associated with anticancer. doi: 10.1016/j.lfs.2003.09.047, Life Sciences, 74, 2157-84 (2004).

Cetin, H.K., y Newman, M.C., Antimicrobial efficacy of plant phenolic compounds against Salmonella and Escherichia Coli. doi: 10.1016/j.fbio.2015.03.002, Food Bioscience,11, 8-16 (2015).

Costa, R.P., Leitao, E.V., y otros cinco autores, Virtual screening of secondary metabolites of the genus Solanum with potential antimicrobial activity. doi: 10.1016/j.bjp.2018.08.003, Revista Brasileira de Farmacognosia, 28(6), 686-691 (2018).

Famuwagun, A. A., Taiwo, K.A., Gbadamosi, S. O; Oyedele, D. J; Aluko, R. E., y Adebooye, O. C, Extraction Optimization and Antioxidant Properties of African Eggplant (Solanum macrocarpon). doi: 10.1155/2017/2159183, Journal of Food Quality, 14 (2017).

Fellegrini, N., Ke, R., Yang, M., y Rice, C.R., Screening of Dietary Carotenoids and Carotenoid-Rich Fruit Extractsf or Antioxidan Activities Applying 2,2'-Azinobis (3-Ethylenebenzothiazoline-6-sulfonic acid) Radical Catión Decolorization Assay. doi: 10.1016/S0076-6879(99)99037-7, Methods in Enzymology, 299, 379-89 (1999).

Gancel, A., Alter, P., Mayer, C.D., Ruales, J; y Vaillant, F; Identifying Carotenoids and Phenolic Compounds in Naranjilla (Solanum quitoense Lam. Var. Puyo Hybrid), an Andean Fruit. doi: 10.1021/jf801515p, Journal of Agricultural and Food Chemistry, 56 (24), 11890-9 (2008).

Ghassam, B.J., Ghaffari, H., Kumara, K., Prakash, H., y Ramachandra, K., Evaluation of the antioxidant and hepatoprotective effect of Solanum grandiflorum Ruiz \& Pav against $\mathrm{CCl}_{4}$-induced oxidative stress in rats. International Journal of Research in Phytochemistry \& Pharmacology, 2(4), 180-7 (2012).

Haddabi, B., Lawati, H.A., y Suliman, F.O., A comprehensive evaluation of three microfluidic chemiluminescence methods for the determination of the total phenolic contents in fruit juices. doi: 10.1016/j.foodchem.2016.07.119, Food Chemistry, 214, 670-7 (2017).

Heras, I., y Alvis, A, Optimización del Proceso de Extracción de Antocianinas y Evaluación de la Capacidad Antioxidante de Berenjena (Solanum melonera L.). doi;10.4067/S0718-07642013000500011, Información Tecnológica, 24(5): 93-102 (2013).

Kaewnarin, K., Suwannarach, N., Kumla, J., y Lumyong, S; Phenolic profile of various wild edible mushroom extracts from Thailand and their antioxidant properties, anti-tyrosinase and hyperglycaemic inhibitory activities. doi:10.1016/j.jff.2016.09.008, Journal of Functional Foods, 27, 352-64 (2016). 
Li, H., Deng, Z., Liu, R., Loewen, S., y Tsao, R., Bioaccessibility, in vitro antioxidant activities and in vivo anti-inflammatory activities of a purple tomato (Solanum lycopersicum L). doi: 10.1016/j.foodchem.2014.03.023, Food Chemistry, 159, 353360 (2014).

Macato, D.R., Monteiro, J.B., y siete autores, Evaluation of Antioxidant Capacity of Solanum sessiliflorum (Cubiu) Extract: An In Vitro Assay. doi: 10.1155/2015/364185, Journal of Nutrition and Metabolism, 1-8 (2015).

Mascherpa, D., Carazzone, C., Marrubini, G; Gazzani, G., y Papetti, A, Identification of Phenolic Constituents in Cichorium endivia Var. crispum and Var. latifolium Salads by High-Performance Liquid Chromatography with Diode Array Detection and Electrospray Ioniziation Tandem Mass Spectrometry. doi: 10.1021/jf3034754, Journal de Agricultural and Food Chemistry, 60, 12142-12150 (2012).

Mora, J.A., Zea, S., y Agudelo, D, Posibles mecanismos químicos y biológicos para el control de la epibiosis de las esponjas Aplysina insularis y Aplysina lacunosa (Demospongiae, Verongida). Acta biológica colombiana, 12, 121-132 (2007).

Muruhan, S., Selvaraj, S., y Viswanathan, P., In vitro antioxidant activities of Solanum surattense leaf extract. doi: 10.1016/S2221-1691(13)60019-2, Asian Pacific Journal of Tropical Biomedicine, 3,28-34 (2013).

Fadl, A., Voynikov, Y., y otros nueve autores, Antibacterial, antiproliferative and antioxidant activity of leaf extracts of selected Solanaceae species. doi: 10.1016/j.sajb.2017.06.016, South African Journal of Botany (112), 368-374 (2017).

Nisha, P., Nazar, A.P; y Jayamurthy, P., A comparative study on antioxidant activities of different varieties of Solanum melongena. doi:10.1016/j.fct.2009.07.026, Food and Chemical Toxicology, 47 (10), 2640-2644 (2009).

Orozco, C., Beltrán, G., Porras, N., y Nee, M., Listado de especies espinosas de Solanum L. (Leptostemonum, Solanaceae), Biota Colombiana, 9(2), 239- 49 (2008).

Pardo, M.A., Efecto in vitro del extracto de Solanum sessiliflorum "cocona" sobre el crecimiento de Helicobacter pylori in vitro effect of Solanum sessiliflorum "cocona" extract on the growth of Helicobacter pylori, Ciencia e Investigación, 13(1), 30-33 (2010).

Patel K., Singh R.B., Patel D.K, Importancia medicinal, actividades farmacológicas y aspectos analíticos de la solasodina: un informe conciso de la literatura científica actual. Revista de enfermedad aguda, 2 (2): $92-98$ (2013).

Rawani, A., Ghosh, A., y Chandra, G, Mosquito larvicidal and antimicrobial activity of synthesized nano-crystalline silver particles using leaves and green berry extract of Solanum nigrum L. (Solanaceae: Solanales). doi: 10.1016/j.actatropica.2013.09.007, Acta Tropical, 128, 613-22 (2013).

Rodrigues, E., Mauriutti, L.R., y Mercadante, A.Z., Carotenoids and phenolic compounds from Solanum sessiliflorum, an unexploited Amazonian fruit, and their scavenging capacities against reactive oxygen and nitrogen species, doi: 10.1021/jf3054214, Journal of Agricultural and Food Chemistry, 61(12), 3022-9 (2013).

Saw, C.L., Guo, Y., Yang, A.Y., y otros cuatro autores, The berry constituent's quercetin, kaempferol, and pterostilbene synergistically attenuate reactive oxygen species: Involvement of the Nrf2-are signaling pathway. doi: 10.1016/j.fct.2014.07.038, Food and Chemical Toxicology, 72, 303-11 (2014).

Siddiqui, N.A., Parvez, M.K., y otros cinco autores, High-performance thin layer chromatography based assay and stress study of a rare steroidal alkaloid solanopubamine in six species of Solanum grown in Saudi Arabia. doi: 10.1016/j.jsps.2016.05.003, Saudi Pharmaceutical Journal, 25(2), 184-195 (2017).

Singleton, V.L., y Rossi, J.A., Colorimetry of total phenolics with phosphomolybdic-phosphotungstic acid reagents. American Journal of Enology and Viticulture, 16, 144-58 (1965).

Xie, P; Huang, L., Zhang, C., y Zhang, Y., Phenolic compositions and antioxidant performance of olive leaf and fruit (Olea europaea L.) extracts and their structure. doi: 10.1016/j.jff.2015.05.005, Journal of Functional Foods, 16, 460-71 (2015).

Williams, W.B., Cuvelier, M.E., y Berset, C., Use of a free radical method to evaluate antioxidant activity. doi: 10.1016/S0023-6438(95)80008-5, Lebensm-Wiss u. Technol, 28(1), 25-30 (1995).

Zadra, M., Piana, M., Brum, F.T., y otros seis autores, Antioxidant activity and phytochemical composition of the leaves of Solanum guaraniticum A. St.-Hil. doi: 10.3390/moléculas171112560, Molecules 17, 12560-12574 (2012).

Zamora, R., y Hidalgo, F.J., The triple defensive barrier of phenolic compounds against the lipid oxidation-induced damage in food products. doi: 10.1016/j.tifs.2016.06.006, Trends in Food Science \& Technology, 54, 165-174 (2016).

Zhishen, J., Mengcheng, T., y Jianming, W., The determination of flavonoid contents in mulberry and their scavenging effects on superoxide radicals. doi: 10.1016/S0308-8146(98)00102-2, Food Chemistry, 64(4), 555-9 (1999). 
